\title{
D-semigroups and constellations
}

\author{
Tim Stokes
}

\begin{abstract}
In a result generalising the Ehresmann-Schein-Nambooripad Theorem relating inverse semigroups to inductive groupoids, Lawson has shown that Ehresmann semigroups correspond to certain types of ordered (small) categories he calls Ehresmann categories. An important special case of this is the correspondence between two-sided restriction semigroups and what Lawson calls inductive categories.

Gould and Hollings obtained a one-sided version of this last result, by establishing a similar correspondence between left restriction semigroups and certain ordered partial algebras they call inductive constellations. (A general constellation is a one-sided generalisation of a category.) We put this one-sided correspondence into a rather broader setting, at its most general involving left congruence D-semigroups (which need not satisfy any semiadequacy condition) and what we call co-restriction constellations, a finitely axiomatized class of partial algebras. There are ordered and unordered versions of our results.

Two special cases have particular interest. One is that the class of left Ehresmann semigroups (the natural one-sided versions of Lawson's Ehresmann semigroups) corresponds to the class of co-restriction constellations satisfying a suitable semiadequacy condition. The other is that the class of ordered left Ehresmann semigroups (which generalise left restriction semigroups and for which semigroups of binary relations equipped with domain operation and the inclusion order are important examples) corresponds to a class of ordered constellations defined by a straightforward weakening of the inductive constellation axioms.
\end{abstract}

Keywords. D-semigroup, left Ehresmann semigroup, constellation.

\section{Introduction}

The Ehresmann-Schein-Nambooripad Theorem (ESN Theorem) may be interpreted as showing that all of the structure of the inverse semigroup $S$ can be recovered by knowing only some of the products in $S$, together with the natural partial order on $S$. Specifically, only two types of product need be retained: those products $x y$ for which $x^{\prime} x=y y^{\prime}$ (where $x^{\prime}$ is the inverse of $x$ ), which by the Vagner-Preston Theorem can be interpreted functionally as saying that the domain of the injective partial function $y$ coincides with the range of $x$; and all products ef where $e, f$ are idempotent, which

\footnotetext{
${ }^{0} \mathrm{~T}$. Stokes

Department of Mathematics, The University of Waikato

Hamilton, New Zealand

email: tim.stokes@waikato.ac.nz
} 
may be interpreted as retaining all information about intersections of domains and ranges. Moreover one can characterise the resulting ordered partial algebras as certain types of small ordered categories called inductive groupoids, which are equipped with restriction and co-restriction operations. Indeed the category of inductive groupoids is isomorphic to the category of inverse semigroups.

In [14], Lawson generalised this result to cover what he called Ehresmann semigroups. Although Lawson did not define them this way, one can view Ehresmann semigroups as bi-unary semigroups in which one has "domain" and "range" operations $D, R$ satisfying a small number of equational laws. Then the set of "domain elements" $D(S)=\{D(s) \mid s \in S\}=\{R(s) \mid s \in S\}=R(S)$ is a subsemigroup of $S$ that is a semilattice on which $D, R$ act trivially. Every inverse semigroup is an Ehresmann semigroup if one defines $D(a)=a a^{\prime}$ and $R(a)=D\left(a^{\prime}\right)=a^{\prime} a$, where $a^{\prime}$ is the inverse of $a$. Also, the semigroup of binary relations $\mathcal{B}(X)$ on a set $X$ is an Ehresmann semigroup in which $D(\rho)$ is the restriction of the diagonal relation (the identity function) to the domain of $\rho$, and $R(\rho)$ is its restriction to the range of $\rho$. (That the laws $D(a b)=D(a D(b))$ and $R(a b)=R(R(a) b)$ hold in $\mathcal{B}(X)$ was probably first noted by Bredikhin in [4]; more recently it was noted in [12] and later again by $[17])$.

Lawson showed that a similar construction to that used for the ESN Theorem allows one to capture the information in the Ehresmann semigroup $S$. One need retain only those products $a \cdot b$ for which $R(a)=D(b)$, together with all products es (resp. se) where $s \in S, e \in D(S)$ and $e \leq D(s)$ (resp. $e \leq R(s)$ ) under the semilattice partial order on $D(S)$, together with two associated partial orders on $S$ induced by left and right multiplication by domain elements, both of which extend $\leq$ on $D(S)$. Again, it is possible to characterise the resulting ordered partial structures as "Ehresmann categories". These are certain types of small categories equipped with two partial orders and two partial operations capturing left and right multiplication by domain elements. Indeed the category of Ehresmann semigroups (in which arrows are semigroup homomorphisms additionally respecting $D, R$ ) is isomorphic to the category of Ehresmann categories.

A special case of the Lawson result arises when $S$ is a two-sided restriction semigroup. In Lawson's terms, this is called an idempotent-connected Ehresmann semigroup, also known in the literature as a weakly E-ample semigroup. Every inverse semigroup is a two-sided restriction semigroup under $D, R$. The category of two-sided restriction semigroups is isomorphic to the category of what Lawson calls inductive categories (by analogy with inductive groupoids). In this case, the two partial orders coincide.

However, at least as important as two-sided restriction semigroups are left restriction semigroups, in which only the unary operation $D$ is defined. For example, the semigroup of partial functions on a set is a left restriction semigroup but not a two-sided restriction semigroup, and indeed all left restriction semigroups embed in such examples. (No such similar interpretation applies to two-sided restriction semigroups.) This representation theorem was first given by Trokhimenko in [20]. Left restriction semigroups are also known as weakly type SL $\gamma$-semigroups in [1], left E-ample semigroups (a term first used in [5] and then again in papers including 
[8]), guarded semigroups (see [16]), and twisted $\mathcal{L} C$-semigroups (see [11] and [12]). It is therefore natual to seek to extend Lawson's results, at least to left restriction semigroups. The problem is that the presence of a domain operation but no range operation in left restriction semigroups means that they do not correspond to a type of category in any natural way.

In [7], Gould and Hollings showed how to define a one-sided version of a category they called a constellation that could be used to capture left restriction semigroups. As for two-sided restriction semigroups, one retains only some of the products in the left restriction semigroup $S$. But the lack of any notion of range means that more products must be retained. Thus, $a \cdot b$ is defined if $a D(b)=a$, corresponding in the partial function interpretation to the notion that the range of $a$ is a subset of the domain of $b$ (rather than equal to it). A more general form of right multiplication by idempotent domain elements must also be retained, and as for two-sided restriction semigroups, only one partial order is needed (that given by $a \leq b$ if $a=e b$ for some $e \in D(S)$ ). The corresponding class of ordered partial algebras is axiomatized in [7] as the class of inductive constellations. An isomorphism between the category of left restriction semigroups and the category of inductive constellations (with suitably defined arrows) is established there.

It is natural to try to generalise the Gould and Hollings result, and to find an appropriate type of partial algebra that corresponds to "left Ehresmann semigroups" (one-sided versions of the Ehresmann semigroups considered by Lawson). Aside from Ehresmann semigroups, examples of these include all binary relations on a set with domain restricted in some way (for example to finite subsets of an infinite set) but with ranges not so restricted. The approach in [7] does not seem capable of generalising to this setting, since a single partial order does not seem sufficient to capture both left and right multiplication by idempotents, whereas retaining two partial orders does not seem to work either because the right-sided partial order given by $a \leq b$ if and only if $a=b e$ for some domain element $e \in D(S)$ is not compatible with the constellation product.

However, even for inductive groupoids, Ehresmann categories and inductive constellations, one can do without the various partial orders altogether. This is because in each case, the partial order may be expressed in terms of the restriction (and corestriction if relevant) partial operation(s) involving domain elements, so one could in theory re-express the axioms without direct reference to partial orders by translating the axioms involving them into first-order sentences involving restriction and co-restriction alone. Indeed one might argue that such an approach is more in the spirit of seeking to keep only some of the products in the original semigroup. Partial orders do not feature in the definitions of the various types of semigroups considered and so need not feature in the definitions of the associated partial algebras either.

This is the approach we take (indeed are forced to take) in the case of left Ehresmann semigroups. As is the case with left restriction semigroups, the associated partial algebras are constellations. But this time the constellation is equipped only with a general notion of multiplication on the right by a domain element (co-restriction), since left-multiplication by idempotents is captured sufficiently well by the constellation product itself. We show that inductive constellations may be viewed as special 
kinds of such constellations with co-restriction, with the partial order and restriction determined by the structure of the constellation.

In fact we do something rather more general. One can generalise left Ehresmann semigroups to general left congruence D-semigroups in the sense of [19]. If $S$ is a general left congruence D-semigroup then $D(S)$ consists of idempotents which need not commute with one-another nor even be closed under multiplication. Such objects arise very naturally, for example as the multiplicative semigroups of Rickart *-rings. (The main example of a Rickart *-ring is all bounded linear operators on a Hilbert space, in which the elements of the form $D(a)$ are orthogonal projections which do not generally commute with one-another. These were first introduced by Maeda in [15], but see also [2] for a comprehensive survey). All our work on the one-sided case goes through at this greater level of generality. Such a generalisation does not seem feasible in the two-sided case considered in [14]. In this setting especially, it seems essential to do without any kind of ordering on the constellations, since the D-semigroups themselves are not naturally ordered.

The natural order on a left restriction semigroup is compatible with both its operations. In the standard example of all partial functions on a set, this partial order is simply set inclusion of (the graphs of) two partial functions. For the left Ehresmann semigroup of binary relations on a set, the partial order of set inclusion is compatible with the operations, although it apparently cannot be expressed in terms of its algebraic structure. This leads to a definition of ordered left Ehresmann semigroups, and more generally, ordered left congruence D-semigroups. With the aid of our earlier results, we are able to characterise those ordered constellations that arise from such ordered unary semigroups. They turn out to be natural generalisations of inductive constellations, with axioms having a similar form. But unlike inductive constellations (and indeed inductive groupoids and Ehresmann categories), the ordering cannot be reduced to algebra: the same constellation can have more than one such ordering. Again, we obtain an isomorphism between the categories of ordered unary semigroups and the corresponding types of small ordered constellations.

Throughout, we use left to right notation for composition of functions, so " $f g$ " denotes "first $f$, then $g$ ".

\section{D-semigroups and constellations}

\section{$2.1 \quad$ D-semigroups}

For a semigroup $S$, let $E(S)$ denote its set of idempotent elements, partially ordered by the standard order given by $e \leq f$ if $e=e f=f e$.

An important example of a constellation is that arising from a left restriction semigroup, and these are characterised in [7] in terms of inductive constellations. However, much more general types of unary semigroups give rise to constellations in a similar way. As in [19], a D-semigroup can be defined to be a unary semigroup $S$ equipped with a "generalised domain" operation $D$ satisfying the following laws: for all $a, b \in S$,

$$
\text { (D1) } D(a) a=a
$$


(D2) $D^{2}(a)=D(a)$

(D3) $D(a b) D(a)=D(a) D(a b)=D(a b)$.

Other laws follow, such as $D(a)^{2}=D(a)$. Note that $D(S)$ need not be closed under multiplication.

It is shown in [19] that a D-semigroup is a semigroup $S$ in which there is $U \subseteq E(S)$ such that, for all $a \in S$, there is a smallest $e \in U$ (with respect to the standard order on $E(S))$ with the property that $e a=a$; this is $D(a)$, and $D(S)=\{D(a) \mid a \in S\}=U$.

In [19], we defined the subset $U \subseteq E(S)$ to be left-reduced if for all $e, f \in U$, $e=f e \Rightarrow e=e f$; in particular, we say a band is left-reduced if it satisfies this law globally. This is a one-sided version of the notion of being reduced, introduced by Lawson in [14]. As in [19], a unary semigroup $S$ is a D-semigroup if and only if $S$ is a left $D(S)$-semiabundant semigroup in which $D(S)$ is left-reduced, as we now explain.

We first define the following generalised Green's relation on the semigroup $S$, in terms of $U \subseteq E(S)$ :

$$
(x, y) \in \widetilde{\mathcal{R}}_{U} \text { if for all } e \in U, e x=x \Leftrightarrow e y=y .
$$

Then $\widetilde{\mathcal{R}}_{U}$ is an equivalence relation. $S$ is said to be left $U$-semiabundant (or weakly left $U$-abundant) if each $\widetilde{\mathcal{R}}_{U}$-class contains at least one element of $U$. If also $U$ is leftreduced, each $\widetilde{\mathcal{R}}_{U}$-class contains a unique element of $U$. If we call this $D(a)$ for any $a$ in that $\widetilde{\mathcal{R}}_{U}$-class, then viewing $D$ as a unary operation gives a D-semigroup, and all Dsemigroups arise in this way. These are the one-sided versions of the $U$-semiabundant semigroups considered by Lawson in [14].

Let $S$ be a D-semigroup. Then $S$ is D-semiadequate if $e f=f e$ for all $e, f \in D(S)$. From this it follows easily that $D(S)$ is a closed under multiplication and hence is a semilattice. In this way we recover the type SL $\gamma$-semigroups of [1], the weakly left Eample semigroups as in [5], the left C-semigroups of [11] and the guarded semigroups of [16]. We say $S$ satisfies the left congruence condition if $D(a b)=D(a D(b))$ for all $a, b$, which is equivalent to $\widetilde{\mathcal{R}}_{U}$ being a left congruence. Because in any D-semigroup, $D(a D(b)) a b=D(a D(b)) a D(b) b=a D(b) b=a b$, we always have $D(a b) \leq D(a D(b))$ by the minimality of $D(a b)$, so the left congruence condition is equivalent to the law $D(a D(b)) \leq D(a b)$.

Definition 2.1 A D-semigroup is left Ehresmann if it is D-semiadequate and satisfies the left congruence condition.

Left Ehresmann D-semigroups are one-sided versions of the Ehresmann semigroups considered in [14]. An example is $(\mathcal{B}(X), \cdot, D)$, the D-semigroup of binary relations on a set equipped with domain operation $D$, where $D(\rho)$ is the restriction of the diagonal relation to the domain of $\rho \in \mathcal{B}(X)$. (Indeed it is an Ehresmann semigroup since there is an analogous range operation as well.) Free left Ehresmann semigroups are described in terms of trees in [13], and (in the monoid case) in terms of a generalised semidirect product construction involving monoids acting as orderpreserving maps on semilattices with identity in the sequence of papers [3] and [6]. 
See [19] for examples of non-D-semiadequate left congruence D-semigroups arising in functional analysis as the multiplicative semigroups of Rickart $*$-rings.

A D-semigroup is a left restriction semigroup if it is D-semiadequate and satisfies $a D(b)=D(a b) a$ for all $a, b$. These are all left Ehresmann, and are shown in [7] to correspond to inductive constellations. As mentioned previously, the main example is $(\mathcal{P}(X), \cdot D)$, consisting of all partial functions on the set $X$ equipped with composition and domain operation.

In a D-semigroup $S$, if $D(S)$ is closed under multiplication, then it is a band. Recall that a band $B$ is said to be left-regular if $e f=e f e$ for all $e, f \in B$.

Lemma 2.2 $A$ band is left-reduced if and only if it is left-regular.

Proof. Suppose the band $B$ is left-reduced. Then for all $e, f \in B,(e f)(e f e)=$ $(e f)^{2} e=e f e$, so $e f=e f e f=(e f e) e f=e f e$, so $B$ is left-regular.

Now suppose $B$ is left-regular. If $e f=f$ for some $e, f \in B$ then $f e=(e f) e=$ $e f=f$, so $B$ is left-reduced.

Corollary 2.3 If $S$ is a D-semigroup in which $D(S)$ is closed under multiplication, then it is a left-regular band.

Every left-regular band arises as $D(S)$ where $S$ is a left congruence D-semigroup, as follows from the next easily verified observation. (The proof is immediate given the fact that Green's relation $\mathcal{R}$ is equality in this case, which is trivially a left congruence.)

Proposition 2.4 Every left-regular band $S$ is a left congruence D-semigroup if we set $D(x)=x$ for all $x \in S$.

\subsection{Constellations}

As in [7], a constellation is a triple $(P, \cdot, D)$ consisting of a set $P$ with a partial binary operation - and unary operation $D$ (denoted ${ }^{+}$in [7]) that maps onto $E \subseteq P$, such that for all $e \in E, e \cdot e$ exists and equals $e$, for all $x, y, z \in P$ :

(C1) if $x \cdot(y \cdot z)$ exists then so does $(x \cdot y) \cdot z$, and then the two are equal;

(C2) $x \cdot(y \cdot z)$ exists if and only if $x \cdot y$ and $y \cdot z$ exist;

(C3) for each $x \in P, D(x)$ is the unique left identity of $x$ in $E$ (i.e. it satisfies $D(x) \cdot x=x)$

(C4) for $a \in P$ and $g \in E$, if $a \cdot g$ exists then it equals $a$.

Note that $E=\{D(a) \mid a \in P\}$ because $D(e)=e$ for all $e \in E$, as is noted early in Section 2 of [7]). Hence, for clarity we here denote $E$ by $D(P)$, which also indicates its association with $P$.

As in [7], which is mainly concerned with inductive constellations and their relationship to left restriction semigroups, it is shown that the class of constellations can 
be made into a category whose arrows are radiants. These are defined to be mappings $\rho: P \rightarrow Q$ (where $P, Q$ are constellations) for which, for all $s, t \in P$,

- if $s \cdot t$ exists in $P$ then $\rho(s) \cdot \rho(t)$ exists in $Q$, with $\rho(s) \cdot \rho(t)=\rho(s \cdot t)$, and

- $\rho(D(s))=D(\rho(s))$.

The radiant is strong if $s \cdot t$ exists in $P$ whenever $\rho(s) \cdot \rho(t)$ exists in $Q$, and an embedding if it is strong and injective. (Use of the word "strong" here is consistent with its usage for partial homomorphisms as in [10].) In [9], the authors consider constellations in full generality, concentating on their relationship with categories.

It is shown in [7] that the class of inductive constellations (constellations equipped with a partial order satisfying certain conditions) is isomorphic as a category to the class of left restriction semigroups. In what follows, we generalise this correspondence by replacing inductive constellations by more general types of constellations on the one hand, and by replacing left restriction semigroups by left congruence D-semigroups on the other.

\subsection{Left congruence D-semigroups give constellations}

Given a D-semigroup $S$, define a new structure $\mathbf{P}(S)=(S, \cdot, D)$ from $S$, having the same underlying set, as follows:

- define the partial binary operation - via $x \cdot y=x y$ if $x D(y)=x$ and undefined otherwise,

- retain the unary operation $D$.

This is the construction for obtaining an inductive constellation from a left restriction semigroup used in [7], except that we have no order structure here. The construction is essentially one-sided and differs from that for obtaining an inductive groupoid from an inverse semigroup as in the ESN Theorem, or indeed that for obtaining an Ehresmann category from an Ehresmann semigroup as in [14].

A two-sided analogue (involving categories and two-sided D-semigroups, appropriately defined) of the following result was observed by Lawson after the proof of Lemma 3.8 in [14].

Proposition 2.5 For a D-semigroup $S$, the following are equivalent:

1. S satisfies the left congruence condition;

2. $S$ satisfies the law $x D(y)=x \Rightarrow D(x y)=D(x)$;

3. $\mathbf{P}(S)$ is a constellation.

Proof. $\quad(1) \Rightarrow(2)$ is obvious.

Suppose (2) holds. We verify $(\mathrm{C} 1)-(\mathrm{C} 4)$ for constellations in turn.

(C1) Suppose $x \cdot(y \cdot z)$ is defined. Then $y D(z)=y$ so $D(y z)=D(y)$, and $x D(y z)=x$. So $x D(y)=x D(y z)=x$, and $x y D(z)=x y$, and so $(x \cdot y) \cdot z$ is defined. As $S$ is a semigroup, in this case it will equal $x \cdot(y \cdot z)$. 
(C2) We just saw that if $x \cdot(y \cdot z)$ is defined then so is $x \cdot y$, and of course so is $y \cdot z$. Conversely, if $x \cdot y$ and $y \cdot z$ are defined, then $x D(y)=x$ and $y D(z)=y$, so $D(y z)=D(y)$, and so $x D(y z)=x D(y)=x$, so $x \cdot(y \cdot z)$ is defined.

(C3) Of course $D(x) D(x)=D(x)$, so $D(x) \cdot x$ exists, and $D(x) \in D(S)$. If also $e \cdot x=x$ for some $e \in D(S)$, then $e D(x)=e$, so $D(e x)=D(e)=e$, and then $D(x)=D(e \cdot x)=D(e x)=e$, showing uniqueness.

(C4) If $a \cdot e$ exists for some $e \in D(S)$, then $a D(e)=a$, and then $a \cdot e=a e=$ $a D(e)=a$. So $(3)$ is established.

Finally, assume (3). Now by (C2), we have $a D(b)=a$ and $b D(c)=b$ imply $a D(b c)=a$ for all $a, b, c \in S$. Let $y, z \in S$ and put $a=D(y D(z)), b=y D(z)$ and $c=z$, giving that $D(y D(z)) D(y D(z))=D(y D(z))$ and $y D(z) D(z)=y D(z)$ (both of which are true!) imply that $D(y D(z)) D(y D(z) z)=D(y D(z))$, that is, $D(y D(z)) D(y z)=D(y D(z))$. But because $D(y z) \leq D(y D(z))$ as noted earlier, we have in particular that $D(y D(z)) D(y z)=D(y z)$. Hence $D(y z)=D(y D(z))$ for all $y, z \in S$, so (1) holds.

\subsection{Inductive constellations}

The following are Definitions 3.1 and 3.3 in [7]. An ordered constellation $(P, \cdot, D, \leq)$ is a constellation $(P, \cdot, D)$ equipped with a partial order $\leq$ such that:

(O1) if $a \leq c$ and $b \leq d$, and both $a \cdot b$ and $c \cdot d$ exist, then $a \cdot b \leq c \cdot d$;

(O2) if $a \leq b$ then $D(a) \leq D(b)$;

(O3) if $e \in D(P)$ and $a \in P$ with $e \leq D(a)$, then there is a unique element $x$ of $P$ for which $x \leq a$ and $D(x)=e$, the restriction of $a$ to $e$, denoted $e \mid a$;

(O4) if $e \in D(P)$ and $a \in P$, then there is a maximum $x \in P$ such that $x \leq a$ and $x \cdot e$ exists, the co-restriction of $a$ to $e$, denoted $a \mid e$;

(O5) for $x, y \in P$ and $e \in D(P)$, if $x \cdot y$ exists then $D((x \cdot y) \mid e)=D(x \mid(D(y \mid e))$;

(O6) if $e, f \in D(P)$ and the restriction $e \mid f$ is defined, then it equals the corresponding co-restriction.

It is inductive if also

(I) every $e, f \in D(P)$ have a greatest lower bound $e \wedge f$ with respect to $\leq$ in $D(P)$, equal to the co-restriction $e \mid f$.

A related property is the following:

$\left(\mathrm{O5}^{\prime}\right)$ for $x, y \in P$ and $e \in D(P)$, if $x \cdot y$ exists then $(x \cdot y) \mid e=(x \mid(D(y \mid e)) \cdot(y \mid e)$.

Because $D(x \cdot y)=D(x)$ whenever $x \cdot y$ exists in a constellation (Lemma 2.2 in [7]), this implies (O5).

It is shown in [7] that the constellation $\mathbf{P}(S)$ arising from a left restriction semigroup $S$ is an inductive constellation in which $a \leq b$ means $a=D(a) b$ for all $a, b \in S$ 
(the natural partial order on $S$ ), and then $e \mid a=e a$ when $e \leq D(a)(a \in S)$, and $a \mid e=a e$ for all $a \in S$ and $e \in D(S)$ (Proposition 4.1 in [7]). Indeed it is shown that left restriction semigroups correspond one-to-one with inductive constellations, and that there is a category isomorphism between the two classes. It is this link that we seek to generalise here. We replace "left restriction semigroup" with "left congruence D-semigroup" and "inductive constellation" with some more general notion. There are ordered and unordered versions of this generalisation, and we begin with the latter.

\section{The natural quasiorder and inductive constellations}

\subsection{The natural quasiorder on a constellation}

Throughout this section, let $P$ be a constellation.

Definition 3.1 The relation $\lesssim$ on $D(P)$ given by $e \lesssim f$ if and only if $e \cdot f$ exists is called the standard quasiorder on $D(P)$.

The above name is appropriate since $\lesssim$ is easily seen to be reflexive and transitive on $D(P)$, hence is a quasiorder. It extends to a quasiorder $\lesssim$ defined on all of $P$.

Definition 3.2 Define the relation $\lesssim$ on $P$ by setting $x \lesssim y$ if $x=e \cdot y$ for some $e \in D(P)$. We call this the natural quasiorder on $P$.

Now $\lesssim$ is indeed a quasiorder: reflexivity of $\lesssim$ is clear (put $e=D(x)$ to see that $x \lesssim x$ ), while for transitivity, if $x=e \cdot y$ and $y=f \cdot z$ for some $x, y, z \in P$ and $e, f \in D(P)$, then $x=e \cdot(f \cdot z)=(e \cdot f) \cdot z=e \cdot z$. Note also that $x \lesssim y$ if and only if $x=D(x) \cdot y$ (since $D(e \cdot y)=D(e)=e$, again by Lemma 2.2 in [7]).

Let $P$ be an inductive constellation as in [7], with given partial order $\leq$. If $a \leq b$ then $a=D(a) \cdot b$ by 3.4 (i) and 3.5 there, so $a \lesssim b$. Conversely, if $a \lesssim b$ then $a=D(a) \cdot b$, so by 2.3 in [7], $D(a) \cdot D(b)=D(a)$ exists, so by 3.2 there, $D(a) \mid D(b)=D(a)$, and (I) gives $D(a) \leq D(b)$, so by (O1), $a=D(a) \cdot b \leq D(b) \cdot b=b$, and so $a \leq b$. So the natural quasiorder on an inductive constellation equals the given partial order on it. In general we have the following.

Proposition 3.3 The natural quasiorder on the constellation $P$ is a quasiorder satisfying (O1), (O2) and (O3) with $e \mid a=e \cdot a$ whenever $e \lesssim D(a)$, and agrees with the standard quasiorder when restricted to $D(P)$.

Conversely, any quasiorder on $P$ that satisfies (O1), (O2) and (O3) and agrees with the standard quasiorder on $D(P)$ is equal to the natural quasiorder (and so $e \mid a=e \cdot a$ whenever $e \lesssim D(a))$.

Proof. To show (O1), suppose $a=e \cdot c$ and $b=f \cdot d$ where $a, b, c, d \in P$ and $e, f \in D(P)$, with $a \cdot b$ and $c \cdot d$ defined. Then since $e \cdot c$ is defined, so is $e \cdot(c \cdot d)$ and 
hence also $(e \cdot c) \cdot d$ which it equals, so that

$$
\begin{aligned}
a \cdot b & =(e \cdot c) \cdot(f \cdot d) \\
& =((e \cdot c) \cdot f) \cdot d \\
& =(e \cdot c) \cdot d \\
& =e \cdot(c \cdot d) .
\end{aligned}
$$

For $(\mathrm{O} 2)$, if $a=e \cdot b$ for some $e \in D(P)$, then $D(a)=D(e \cdot b)=D(e)=e \lesssim D(b)$ since $e \cdot D(b)$ exists.

For (O3), if $e \in D(P)$ and $a \in P$ with $e \lesssim D(a)$, then $e=e \cdot D(a)$, so $x=e \cdot a$ exists. Then $x \lesssim a$, and $D(x)=D(e)=e$. Moreover if $y \in P$ is such that $y \lesssim a$ and $D(y)=e$, then $y=f \cdot a$ for some $f \in D(P)$ and so $D(y)=D(f)=f=e$, so $y=x$. So $x$ has the required properties for $e \mid a$.

Finally, if $e, f \in D(P)$, then $e \lesssim f$ in $P$ if and only if $e=D(e) \cdot f=e \cdot f$, that is, $e \lesssim f$ under the standard quasiorder on $D(P)$.

Now suppose $\leq$ is a quasiorder on $P$ satisfying (O1) and (O3) and agreeing with $\lesssim$ on $D(P)$, in which case (O2) holds automatically as well. Then suppose $a \leq b$. Let $e=D(a) \leq D(b)$; then $a=e \mid b$ by (O3). Also, $e \leq D(b)$, so $e \cdot D(b)$ exists, and so $e \cdot b$ does, and $D(e \cdot b)=e$ with $e \cdot b \lesssim D(b) \cdot b=b$, so also $e \mid b=e \cdot b$. So $a=e \cdot b$ and $a \lesssim b$. Conversely, if $a \lesssim b$, so that $a=D(a) \cdot b$, then $D(a) \cdot D(b)$ exists, giving $D(a) \lesssim D(b)$, and so $a=D(a) \cdot b \leq D(b) \cdot b=b$. So $\leq$ and $\lesssim$ coincide on all of $P$.

It is straightforward to decide when the natural quasiorder is a partial order.

Proposition 3.4 For the constellation $P$, the following are equivalent.

1. The natural quasiorder is a partial order.

2. The standard quasiorder on $D(P)$ is a partial order.

3. For all $e, f \in D(P)$, if $e \cdot f$ and $f \cdot e$ exist then $e=f$.

Proof. Obviously $(1) \Rightarrow(2)$. Conversely, if the standard quasiorder on $D(P)$ is a partial order and $a \lesssim b$ and $b \lesssim a$, so that $a=e \cdot b$ and $b=f \cdot a$ for some $e, f \in D(P)$, then $a=e \cdot(f \cdot a)=(e \cdot f) \cdot a$, so $e \cdot f$ exists. Similarly $f \cdot e$ exists, so $e \lesssim f$ and $f \lesssim e$, giving $e=f$. But certainly $e=D(a)$, and so we get $b=f \cdot a=e \cdot a=D(a) \cdot a=a$. This shows that the natural quasiorder is antisymmetric, hence a partial order, and so $(2) \Rightarrow(1)$.

The equivalence of (2) and (3) is obvious.

\subsection{The case of inductive constellations}

We next show that the notion of an inductive constellation can be formulated in a straightforward order-free manner.

Lemma 3.5 If the natural quasiorder in a constellation satisfies (O4), then it is a partial order, and (O6) and (I) are also satisfied. 
Proof. Suppose (O4) holds for $\lesssim$. Then for $e, f \in D(P)$, there is a largest $x \in P$ (namely the co-restriction $e \mid f$ ) for which $x \lesssim e$ and $x \cdot f$ exists. But since $x \lesssim e$, we have $x=D(x) \cdot e=D(x)$, so $x \in D(P)$. So $x \cdot f$ exists simply asserts that $x \lesssim f$ in $D(P)$, showing that $x$ must be the greatest lower bound of $e, f \in D(P)$. So (I) holds.

If $e \lesssim f$ and $f \lesssim e$ (where $e, f \in D(P)$ ), then $e$ and $f$ are both maximal lower bounds of $e, f$, hence are equal by (I). So the restriction of $\lesssim$ to $D(P)$ is a partial order on $D(P)$, hence $\lesssim$ is a partial order on $P$ by Proposition 3.4.

If $e, f \in D(P)$ and the restriction $e \mid f$ exists, it is $e \cdot f=e$ by Proposition 3.3, and so $e \lesssim f$. Then the co-restriction $e \mid f$ is their greatest lower bound, which is $e$, so the restriction and co-restriction coincide, establishing (O6).

In view of Proposition 3.3 and the remark preceding it, we have the following.

Corollary 3.6 A constellation is inductive with respect to its natural quasiorder $\leq$ if and only if there is a co-restriction satisfying (O4) and (O5) with respect to $\leq$.

So in particular, if $P$ is an ordered constellation with respect to its natural quasiorder, then it automatically satisfies (I) and so is inductive.

\section{D-semigroups and constellations with co-restriction}

In [7], it is shown that inductive constellations arise from left restriction semigroups using the construction $S \mapsto \mathbf{P}(S)$ as described in Section 2.3. Conversely, it is shown that there is a construction $P \mapsto \mathbf{T}(P)$ that converts inductive constellations into left restriction semigroups. Moreover these two constructions are shown to be mutually inverse. After showing also that morphisms correspond, it follows that the categories of left restriction semigroups and inductive constellations are isomorphic. Our goal in this section is to generalise this correspondence. We begin by showing that in the definition of inductive constellations, the partial order and the restriction notions are not necessary, by giving sufficiently many axioms for co-restriction without reference to orderings at all.

Proposition 4.1 A constellation $P$ is inductive with respect to its natural quasiorder if and only if it is equipped with an operation $\mid: P \times D(P) \rightarrow P$, mapping $(a, e)$ to $a \mid e$, satisfying (O5) and the following:

- if e, $f \in D(P)$ are such that $e \cdot f$ and $f \cdot e$ exist then $e=f$, and

- for all $a \in P$ and $e, f \in D(P),(f \cdot a) \cdot e$ exists if and only if $f \cdot(a \mid e)$ exists, and then they are equal.

Then when $P$ is viewed as an inductive constellation, the given partial order on $P$ is the natural quasiorder, $e \mid a=e \cdot a$ whenever $e \lesssim D(a)$ in $D(P)$ is the restriction operation on $P$, and a|e is co-restriction.

Proof. If $P$ is inductive, we know from the remarks prior to Proposition 3.3 that the natural quasiorder $\lesssim$ on $P$ is its given partial order, hence antisymmetric, so the first law above holds. For $a \in P$ and $e \in D(P), a \mid e=D(a \mid e) \cdot a$ by Lemma 3.6 in [7]. 
Suppose $(f \cdot a) \cdot e$ exists. Since $f \cdot a \lesssim a$, by definition it must be the case that $f \cdot a \lesssim a \mid e$, so $f \cdot a=D(f \cdot a) \cdot(a \mid e)=f \cdot(a \mid e)$, which therefore exists. So in this case, $(f \cdot a) \cdot e=f \cdot a=f \cdot(a \mid e)$.

Conversely, suppose $f \cdot(a \mid e)$ exists. So $f \cdot((a \mid e) \cdot e)$ exists, so $(f \cdot(a \mid e)) \cdot e$ exists, so $(f \cdot(D(a \mid e) \cdot a)) \cdot e$ exists, and so $(f \cdot a) \cdot e=((f \cdot D(a \mid e)) \cdot a) \cdot e$ does.

It follows that $f \cdot(a \mid e)$ exists if and only if $(f \cdot a) \cdot e$ exists, and then as noted above, they are equal.

Conversely, assume the above laws. By Proposition 3.3, the natural quasiorder satisfies (O1) to (O3) with restriction as described, and is a partial order by Proposition 3.4. Next, for $a \in P$ and $e \in D(P)$, we have $D(a \mid e) \cdot(a \mid e)$ exists. So it equals $(D(a \mid e) \cdot a) \cdot e=D(a \mid e) \cdot a \lesssim a$, so $a \mid e \lesssim a$. If $x \lesssim a$ with $x \cdot e$ existing then $x=f \cdot a$ for some $f \in D(P)$ and so $(f \cdot a) \cdot e$ exists and hence equals $f \cdot(a \mid e)$. So $f \lesssim D(a \mid e)$, and $x=f \cdot a=(f \cdot a) \cdot e=f \cdot(a \mid e)$ by hypothesis, so that $x \lesssim a \mid e$. This proves $(\mathrm{O} 4)$ is satisfied. Now apply Corollary 3.6.

This result provides the key insight to generalising the correspondence between left restriction semigroups and inductive constellations to cover general D-semigroups. This is because there seems no useful (quasi-)order-theoretic description of the class of constellations arising from D-semigroups, even left Ehresmann semigroups. This approach also provides a platform from which to build more direct generalisations of inductive constellations in which a quasiorder is present, covered in the final section.

Definition 4.2 A co-restriction constellation is a constellation $P$ equipped with an operation of co-restriction $\mid: P \times D(P) \rightarrow P$, satisfying the following laws: for all $s, t \in S$ and $e, f, g \in D(P)$,

(R1) $s \cdot e$ exists if and only if $s \mid e=s$

(R2) $e \mid f=f$ implies $f \mid e=f$

(R3) if e $\mid f=f$ then $(s \mid e)|f=s| f$

(R4) $D(s) \mid D(s \mid e)=D(s \mid e)$

(R5) if $s \cdot t$ exists then $(s \cdot t) \mid e=(s \mid(D(t \mid e)) \cdot(t \mid e)$.

Moreover $P$ is

- band-like if the co-restriction e|f $f \in D(P)$ for all $e, f \in D(P)$

- D-semiadequate if e $f=f \mid e \in D(P)$ for all $e, f \in D(P)$

- D-ample if it is D-semiadequate and satisfies $s \mid e=D(s \mid e) \cdot s$ for all $s \in S$ and $e \in D(P)$.

Note that restriction can be defined via $e \mid a=e \cdot a$, and (O1) to (O3) will be satisfied as in Proposition 3.3. Law (R2) is obviously redundant in the D-semiadequate case. Also, (R5) is nothing but (O5'), which is in general stronger than (O5). (But note that $\left(\mathrm{O}^{\prime}\right)$ and $(\mathrm{O} 5)$ are equivalent in the inductive constellation setting, as follows from Lemma 3.7 in [7]).

The proof of the following is a routine verification. 
Proposition 4.3 If $S$ is a left congruence D-semigroup then $\mathbf{P}(S)$ is a co-restriction constellation, with co-restriction defined by $s \mid e=$ se for all $s \in S$ and $e \in D(S)$.

If $D(S)$ is a band then $\mathbf{P}(S)$ is band-like, if $S$ is left Ehresmann then $\mathbf{P}(S)$ is $D$-semiadequate, and if $S$ is left restriction then $\mathbf{P}(S)$ is D-ample.

Lemma 4.4 If $P$ is a co-restriction constellation, then for all $e \in D(P)$ and $s \in P$, $e \mid e=e$ and $(s \mid e) \cdot e$ exists.

Proof. For all $e \in D(P), e \cdot e$ exists, so by (R1), $e \mid e=e$. So by (R3), $(s \mid e)|e=s| e$, and so again by $(\mathrm{R} 1),(s \mid e) \cdot e$ exists.

Proposition 4.5 Every co-restriction constellation $P$ determines a left congruence $D$-semigroup $\mathbf{T}(P)$ with multiplication given by $x \otimes y:=(x \mid D(y)) \cdot y$ and with domain operation $D$ the same as in $P$.

If $P$ is band-like then $D(\mathbf{T}(P))$ is a band, if $P$ is D-semiadequate then $\mathbf{T}(P)$ is left Ehresmann, and if $P$ is D-ample then $\mathbf{T}(P)$ is left restriction.

Proof. Note first that $\otimes$ is everywhere-defined. We show it is associative. For all $a, b, c \in S$,

$$
\begin{aligned}
a \otimes(b \otimes c) & =a \otimes((b \mid D(c)) \cdot c) \\
& =[a \mid(D(b \mid D(c)) \cdot c)] \cdot[(b \mid D(c)) \cdot c] \\
& =[(a \mid D(b \mid D(c)) \cdot(b \mid D(c))] \cdot c \text { by }(\mathrm{C} 1)
\end{aligned}
$$

whereas $(a \otimes b) \otimes c=((a \mid D(b)) \cdot b) \otimes c=(((a \mid D(b) \cdot b) \mid D(c)) \cdot c$. So it suffices to show that

$$
(a \mid D(b \mid D(c))) \cdot(b \mid D(c))=(a \mid D(b) \cdot b) \mid D(c) .
$$

But $(a \mid D(b) \cdot b) \mid D(c)=((a \mid D(b)) \mid D(b \mid D(c)) \cdot(b \mid D(c))$ by (R5). Also, $D(b) \mid D(b \mid D(c))=$ $D(b \mid D(c))$ by (R4), so by (R3), $(a \mid D(b)) \mid(D(b \mid D(c))=a \mid D(b \mid D(c))$, and so $((a \mid D(b)$. $b) \mid D(c)=(a \mid D(b \mid D(c))) \cdot(b \mid D(c))$. and

Clearly $D(a) \otimes a=(D(a) \mid D(a)) \cdot a=D(a) \cdot a=a, D^{2}(a)=D(a)$ for all $a \in S$,

$$
D(a \otimes b)=D((a \mid D(b)) \cdot b)=D(a \mid D(b))=D((a \mid D(b)) \cdot D(b))=D(a \otimes D(b))
$$

for all $a, b \in S$. Finally, note that for $e, f \in D(P), e \otimes f=(e \mid D(f)) \cdot f=(e \mid f) \cdot f=e \mid f$, and so for all $a, b \in S$,

$$
\begin{aligned}
D(a) \otimes D(a \otimes b) & =D(a) \mid D(a \otimes b) \\
& =D(a) \mid D((a \mid D(b)) \cdot b) \\
& =D(a) \mid D((a \mid D(b)) \\
& =D(a \mid D(b)) \text { using (R4) } \\
& =D(a \mid D(b)) \cdot b) \text { by Lemma } 4.4 \\
& =D(a \otimes b),
\end{aligned}
$$


so $D(a) \mid D(a \otimes b)=D(a \otimes b)$, and so $D(a \otimes b) \otimes D(a)=D(a \otimes b) \mid D(a)=D(a \otimes b)$ by (R2). Hence $\mathbf{T}(P)$ is a left congruence D-semigroup.

If $P$ is band-like then $e \otimes f=e \mid f \in D(P)=D(\mathbf{T}(P))$, so $D(\mathbf{T}(P))$ is a band.

If $P$ is D-semiadequate, then $e \otimes f=e|f=f| e=f \otimes e$, so $\mathbf{T}(P)$ is left Ehresmann.

If $P$ is D-ample then $a \otimes D(b)=(a|D(D(b)) \cdot D(b)=(a \mid D(b)) \cdot D(b)=a| D(b)=$ $D(a \mid D(b)) \cdot a$. So $D(a \mid D(b)) \cdot D(a)$ exists, and so $(D(a \mid D(b))) \mid D(a)=D(a \mid D(b))$ by (R1). Continuing,

$a \otimes D(b)=(D(a \mid D(b)) \mid D(a)) \cdot a=D(a \mid D(b)) \otimes a=D((a \mid D(b)) \cdot b) \otimes a=D(a \otimes b) \otimes a$, and so $\mathbf{T}(P)$ is a left restriction semigroup.

The operation $\otimes$ on $P$ is defined in terms of the partial constellation operation by making use of co-restriction to ensure that an element with "small enough image", namely $x \mid D(y)$, is used so that the partial product $(x \mid D(y)) \cdot y$ makes sense. Establishing the various laws is then a matter of making appropriate use of laws for co-restriction constellations. In fact the proof is along similar lines to that of Proposition 4.5 in [7], except that sometimes axioms from (R1) to (R5) must be used rather than corresponding lemmas.

Proposition 4.6 If $S$ is a left congruence D-semigroup and $P$ a co-restriction constellation, then $\mathbf{T}(\mathbf{P}(S))=S$ and $\mathbf{P}(\mathbf{T}(P))=P$.

Proof. The proof is formally the same as the proof of Proposition 4.6 in [7], except that sometimes axioms from (R1) to (R5) must be used rather than corresponding lemmas.

These results show that there is a one-to-one correspondence between left congruence D-semigroups and co-restriction constellations. To obtain an isomorphism of categories, we need to consider morphisms as well. For D-semigroups, these will be semigroup homomorphisms that additionally respect $D$. For co-restriction constellations, they are certain types of radiants.

Definition $4.7 A$ co-radiant $\rho: P \rightarrow Q$ between co-restriction constellations is a radiant that additionally satisfies $\rho(s \mid e)=\rho(s) \mid \rho(e)$ for all $s \in P$ and $e \in D(P)$.

Being a co-radiant in the above sense is one of the conditions on a radiant that ensures it is an ordered radiant between ordered constellations in the sense of [7], the other being that $s \leq t$ implies $\rho(s) \leq \rho(t)$. In the inductive constellation case, this other condition holds automatically because the given partial order is the standard order when restricted to $D(P)$. So co-radiants between co-restriction constellations are nothing but ordered radiants when they are between inductive constellations.

If $\rho_{1}: P \rightarrow Q$ and $\rho_{2}: Q \rightarrow R$ are co-radiants between co-restriction constellations $P, Q, R$, then for all $e \in D(P)$ and $s \in P$,

$$
\rho_{2}\left(\rho_{1}(e \mid s)\right)=\rho_{2}\left(\rho_{1}(e) \mid \rho_{1}(s)\right)=\rho_{2}\left(\rho_{1}(e)\right) \mid \rho_{2}\left(\rho_{1}(s)\right) .
$$

So the composite of two co-radiants is a co-radiant, and so we obtain a category of co-restriction constellations in which co-radiants are the morphisms.

The proof of the following is very similar to that of Theorem 4.13 in [7]. 
Theorem 4.8 The category of left congruence D-semigroups is isomorphic to the category of co-restriction constellations and co-radiants.

Proof. As noted, Propositions 4.3, 4.5 and 4.6 establish a one-to-one correspondence between the objects of these two categories. It remains to show that morphisms correspond.

If $\rho: P \rightarrow Q$ is a co-radiant between the co-restriction constellations $P, Q$, then let $\rho_{\mathbf{T}}: \mathbf{T}(P) \rightarrow \mathbf{T}(Q)$ be the same function on the underlying sets. The proof that $\rho_{\mathbf{T}}$ is a D-semigroup homomorphism is easy, and is formally identical to the proofs of Lemma 4.9 and Proposition 4.10 in [7].

Conversely, if $\phi: S \rightarrow T$ is a D-semigroup homomorphism (a semigroup homomorphism respecting $D)$, let $\phi_{\mathbf{P}}: \mathbf{P}(s) \rightarrow \mathbf{P}(T)$ be the same function on the underlying sets. Again the proof that $\phi_{\mathbf{P}}$ is formally identical to the proof of Proposition 4.11 in [7].

Finally, for $\rho: P \rightarrow Q$ a co-radiant between the co-restriction constellations $P, Q$, let $\mathbf{T}(\rho)=\rho_{\mathbf{T}}$, and for $\phi: S \rightarrow T$ a D-semigroup homomorphism, let $\mathbf{P}(\phi)=\phi_{\mathbf{P}}$. It is now clear that $\mathbf{P}(\mathbf{T}(\rho))=\rho$ and $\mathbf{T}(\mathbf{P}(\phi))=\phi$, and so the two categories in the statement of the theorem are indeed isomorphic.

As special cases, we have the following.

Corollary 4.9 The category of left congruence D-semigroups in which $D(S)$ is a band is isomorphic to the category of band-like co-restriction constellations and co-radiants.

The category of left Ehresmann D-semigroups is isomorphic to the category of $D$-semiadequate co-restriction constellations and co-radiants.

The category of left restriction semigroups is isomorphic to the category of Dample co-restriction constellations and co-radiants.

The second paragraph in Corollary 4.9 may be viewed as a one-sided (order-free) analog of Theorem 4.24 in [14]. However, there seems no analog of the first paragraph, involving a correspondence between two-sided D-semigroups and categories. This is because the arguments given by Lawson in [14] make heavy use of the property of D-semiadequacy (that is, the condition that domain elements commute with oneanother).

The final case in Corollary 4.9 indicates that inductive constellations are simply Dample co-restriction constellations (with partial order given by the natural quasiorder, and restriction $e \mid a$ defined to be $e \cdot a$ whenever $e \cdot D(a)$ exists), a slightly different formulation to that given in Proposition 4.1.

In particular, it follows from Corollary 4.9 that the D-semiadequacy property of a co-restriction constellation implies the band-like property: if the D-semigroup $\mathbf{T}(P)$ as in Proposition 4.5 satisfies $e \otimes f=f \otimes e$ for all $e, f \in D(P)=D(\mathbf{T}(P))$, then as noted earlier, it follows easily that $D(\mathbf{T}(P))$ is a semilattice, hence the band-like property holds in $P=\mathbf{P}(\mathbf{T}(P))$. 


\section{$5 \quad$ Restoring order}

Left Ehresmann D-semigroups generalise left restriction semigroups. On a left Ehresmann D-semigroup $S$, we may define the "natural" partial order given by $a \leq b$ if $a=e b$ for some $e \in D(S)$. However, this does not behave especially nicely, since it need not be compatible with multiplication. But natural examples of left Ehresmann semigroups often come equipped with some ordering with respect to which multiplication is compatible. For example, consider $S=\mathcal{B}(X)$, the left Ehresmann D-semigroup of binary relations on the non-empty set $X$ under composition and domain. This possesses its fundamental order, namely set inclusion, which is compatible with its composition and domain operations and agrees with the standard order on $D(S)$. The fundamental order is in general coarser than its natural order (or the obvious right-sided version of it, or even their intersection, none of which is compatible with multiplication); for example, this is the case for $\mathcal{B}(X)$.

In $\mathcal{P}(X)$, the left restriction semigroup of all partial maps on $X$, the fundamental order of set inclusion coincides with the natural partial order. So another way to generalise the left restriction semigroup/inductive constellation correespondence is to try to link suitably ordered constellations to suitably ordered D-semigroups.

\section{$5.1 \quad$ Ordered D-semigroups}

Definition 5.1 A D-semigroup $S$ is ordered if it possesses a partial order $\leq$ that agrees with the standard order on $D(S)$ and satisfies the following laws: for all $a, b, c, d \in S$ and $e \in D(S)$,

$$
a \leq b, c \leq d \text { imply } a c \leq b d \text { and } D(a) \leq D(b) ; \text { and } a e \leq a .
$$

If $S$ in the above is a monoid, then the final condition is equivalent (in the presence of the others) to $e \leq 1$ for all $e \in D(S)$, whence also $e a \leq a$ for all $a \in S$ and $e \in D(S)$. Similarly, if $S$ is D-semiadequate, or more generally if $D(S)$ is a band, then $e a=(e D(a)) a \leq D(a) a=a$ for all $e \in D(S)$ and $a \in S$, since $e D(a) \in D(S)$ and $e D(a) \leq D(a)$ since $\leq$ agrees with the standard order on $D(S)$.

The class of ordered D-semigroups (resp. ordered left congruence D-semigroups) is a category in which morphisms are order-preserving homomorphisms.

The left Ehresmann D-semigroup $\mathcal{B}(X)$ of binary relations on the set $X$ is ordered with respect to inclusion. For an example of an ordered D-semigroup that is not Dsemiadequate, we mention left zero bands in which the partial order is equality. This example can be generalised to certain types of bands as follows.

Recall that a left-regular band $B$ is left-reduced. It follows that the relation $\leq$ given by $e \leq f$ whenever $e=f e$ is nothing but the partial order on $B$ given by $e \leq f$ if and only if $e=e f=f e$. However, in general it need not give an ordered semigroup, since it may not be compatible with multiplication. Now $\leq$ is exactly the quasiorder $\leq_{\mathcal{R}}$ on $B$ determined by Green's relation $\mathcal{R}$ on $B$ (since $a \leq_{\mathcal{R}} b$ if and only if $a B \subseteq b B$, in turn equivalent to $b a=a)$, which is well known to be left compatible with multiplication. (To see this directly, if $e \leq f$ then $e=f e$, so 
$(g f)(g e)=(g f g) e=g f e=g e$, so $g e \leq g f$.$) So to ensure monotonicity, we must$ impose the quasi-identity

$$
e=f e \Rightarrow e g=f g e g,
$$

which is at least satisfied by all semilattices and all left zero semigroups. We have now established the following.

Proposition 5.2 A left-regular band B satisfying the above quasi-identity is an ordered D-semigroup if we define the D-semigroup structure as in Proposition 2.4 (i.e. $D(x)=x$ for all $x \in B$ ).

We mention also the supported quantales considered in [18]. These are quantales, hence semigroups, with a domain-like operation. By their definition as well as Lemmas 3.3 and 3.4 there, these can be shown to be ordered D-semigroups under this domainlike operation and their given order if and only if they are stably supported in the sense given in [18], and then they are also D-semiadequate and have the left congruence property.

In particular, the D-semigroup $\mathcal{B}(X)$ of binary relations on the set $X$ is such a stably supported quantale, hence an ordered D-semiadequate left congruence Dsemigroup. For an example of the latter which is not a stably supported quantale, we need look no further than $\mathcal{P}(X)$, the left restriction semigroup of partial maps on the set $X$, ordered by its inclusion order (in this case definable via $a \leq b$ if and only if $a=D(a) b)$. Although $\mathcal{P}(X)$ has meets under this partial order (intersection), it does not have joins (in particular, the union of partial functions is not in general a partial function), so it is not a quantale. Moreover, it has no involution.

\subsection{Weakly ordered constellations}

We shall be interested in the following further generalisations of the some of the laws of inductive constellations:

(O) for all $e, f \in D(P), e \leq f$ implies $e \cdot f$ exists.

$\left(\mathrm{O}^{\prime}\right)$ if $e \in D(P)$ and $a \in P$ with $e \leq D(a)$, then there is a largest element $x$ of $P$ for which $x \leq a$ and $D(x)=e$, the restriction of $a$ to $e$, denoted $e \mid a$.

Condition $\left(\mathrm{O}^{\prime}\right)$ is a weakening of (O3), and is essentially the left half of condition (OC6) in Lemma 2.6 of [14]. Condition (O) holds for inductive constellations; see Lemma 3.4 (iii) in [7].

Definition 5.3 A constellation $P$ equipped with a partial order $\leq$ is weakly ordered if (O1), (O2), (O3'), (O4), (O5') and (O6) all hold.

In the definition of ordered constellations as in [7], law (O5) may be replaced by $\left(\mathrm{O} 5^{\prime}\right)$; the latter immediately implies the former, and $\left(\mathrm{O} 5^{\prime}\right)$ holds in all ordered constellations by Lemma 3.7 in [7]. Note also that many of the results to follow in this section apply without the need to assume $\left(\mathrm{O} 5^{\prime}\right)$, but since (O5) is part of the definition of ordered constellations given in [7], for the sake of consistency we include $\left(\mathrm{O} 5^{\prime}\right)$ in 
the definition of weakly ordered constellations. The weakly ordered constellation laws are otherwise the same as the ordered constellation laws except that (O3) is replaced by the weaker $\left(\mathrm{O} 3^{\prime}\right)$.

In fact we do not require restriction and the two axioms that refer to it in the definition of weakly ordered constellations, providing we include the simpler law $(\mathrm{O})$.

Proposition 5.4 A constellation $P$ equipped with a partial order $\leq$ is weakly ordered if and only if it satisfies (O), (O1), (O2), (O4) and (O5'), and then $e \mid a=e \cdot a$ for all $e \in D(P)$ for which $e \leq D(a)$.

Proof. Assume $P$ is weakly ordered. Suppose $e \leq f$ where $e, f \in D(P)$. Then the co-restriction $e \mid f$ is also a restriction since $e \leq D(f)=f$. Now $D(e)=e$ and $e \leq f$, so $e \leq e \mid f$ as a restriction. But as a co-restriction, we have $e \mid f \leq e$, so $e=e \mid f$. So because $(e \mid f) \cdot f$ exists, so must $e \cdot f$. So $(\mathrm{O})$ is satisfied.

Conversely, suppose $(\mathrm{O}),(\mathrm{O} 1),(\mathrm{O} 2)$, and $(\mathrm{O} 4)$ are satisfied. First, suppose $e \leq$ $D(a)$, so $e \cdot D(a)$ exists by the assumption that $(\mathrm{O})$ holds. So $e \cdot a$ exists by Lemma 2.3 of [7]. Then we have $D(e \cdot a)=D(e)=e$ and $e \cdot a \leq D(a) \cdot a=a$. If also $D(x)=e$ and $x \leq a$ then $x=D(x) \cdot x=e \cdot x \leq e \cdot a$, so $e \cdot a$ is the largest such $x$, and we may put $e \mid a=e \cdot a$. Hence $\left(\mathrm{O}^{\prime}\right)$ follows and $e \mid a$ is as described. Next suppose $e, f \in D(P)$ and the restriction $e \mid f$ exists, so that $e \leq D(f)=f$. Now $e \cdot f$ exists by $(\mathrm{O})$ and equals $e$; but this is the restriction $e \mid f$ by what was just shown. Also, $e \leq e$ and $e \cdot f$ exists, and amongst those $x$ for which $x \leq e$ and $x \cdot f$ exists, $e$ is obviously the largest. So $e=e \mid f$ as a co-restriction also. So (O6) holds.

Proposition 5.5 In a weakly ordered constellation $P$, the following hold for all $a, b, c \in P$ and $e, f \in D(P)$.

1. The constellation product $a \cdot b$ exists if and only if $a \mid D(b)=a$.

2. If $e \leq f$ then $e \cdot f$ exists and $e=e \mid f=e \cdot f$.

3. If $f \leq e$ then $(a \mid e)|f=a| f$ and $a|f \leq a| e$.

4. If $a \leq b$ then $a|e \leq b| e$.

Proof. These correspond to Lemma 3.2, as well as Lemma 3.4 parts (iii), (v), and (vi) in [7] respectively. For the first claim, the argument is identical to that given in [7]. For the second, if $e \leq f$, then arguing as in the proof of Proposition 5.4, e equals the co-restriction $e \mid f$. The proofs of the third and fourth claims are exactly as for their precursors in [7].

\subsection{Weakly inductive and pre-inductive constellations}

Definition 5.6 A constellation is weakly inductive if it is weakly ordered and satisfies (I), and pre-inductive if it is weakly ordered and satisfies

$$
\text { (I') for all } e, f \in D(P), e \leq f \text { implies } e=e|f=f| e \text { as co-restrictions. }
$$


It is easy to see that (I) implies ( $\left.\mathrm{I}^{\prime}\right)$ and that if (I) holds then $D(P)$ is a semilattice under the given partial order.

From Proposition 5.4, it follows that the laws for pre-inductive constellations that refer to restriction (namely $\left(\mathrm{O}^{\prime}\right)$ and $(\mathrm{O} 6)$ ) are not necessary providing $(\mathrm{O})$ is assumed. (Note that this is not so for the inductive case because (O3) involves a uniqueness claim. On the other hand, the order itself can be dispensed with in the inductive case, as we saw in Proposition 4.1.)

We have the following immediate corollary to Proposition 5.4.

Corollary 5.7 The constellation $P$ is pre-inductive if and only if it satisfies (O), (O1), (O2), (O4), (O5') and ( $\left.I^{\prime}\right)$, and then the restriction ela is e $\cdot a$. The constellation $P$ is weakly inductive if and only if it satisfies (O), (O1), (O2), (O4), (O5') and (I).

Ordered D-semigroups provide the main examples of interest. Recall the definition of $\mathbf{P}(S)$ given at the beginning of Section 2.3.

Proposition 5.8 Let $S$ be an ordered left congruence D-semigroup. Then $\mathbf{P}(S)$ is pre-inductive, with the partial order as on $S$ and restriction given by $e \mid a=e$ for alle for which $e \leq D(a)$, and co-restriction given by a|e $=$ ae, for all $a \in S$ and $e \in D(S)$. If $S$ is D-semiadequate then $\mathbf{P}(S)$ is weakly inductive. If $D(S)$ is a band then $\mathbf{P}(S)$ is band-like.

Proof. Of course $\mathbf{P}(S)$ is a constellation by Proposition 2.5, and (O1) and (O2) are immediate from Definition 5.1. If $e, f \in D(S)=D(\mathbf{P}(S))$ with $e \leq f$ then $e=e f=f e$, so $e \cdot f$ exists, verifying $(\mathrm{O})$.

Let $x=a e$, so that $x \leq a$ and $(a e) e=a e$, and so $x \cdot e$ exists. If $y \leq a$ with $y \cdot e$ existing, then $y=y e \leq a e=x$, so $x$ is the largest such, verifying $(\mathrm{O} 4)$ with $a \mid e=a e$. For $\left(\mathrm{O} 5^{\prime}\right)$, if $x \cdot y$ exists then $(x \mid D(y \mid e)) \cdot D(y \mid e)$ exists whence so does $(x \mid D(y \mid e)) \cdot(y \mid e)=$ $x D(y e)(y e)=x y e=(x \cdot y) \mid e$. So by Proposition 5.4 , the constellation $S$ is weakly ordered. Moreover if $e \leq D(a)$ then $e \mid a=e \cdot a=e a$.

Let $e, f \in D(S)$ with $e \leq f$. Then $e=e f=f e$ so $e=e|f=f| e$, establishing $\left(\mathrm{I}^{\prime}\right)$. Hence $S$ is pre-inductive by Corollary 5.7.

If $S$ is D-semiadequate, then $e|f=e f=f e=f| e$ is the meet of $e, f \in E$, showing (I). If $D(S)$ is a band then $e \mid f=e f \in D(S)$ for all $e, f \in D(S)$.

Note that a left-regular band $B$ satisfying the law $f e=e \Rightarrow f g e g=e g$, made into an ordered D-semigroup as in Proposition 2.4, is left congruence as noted there, and gives rise to a band-like pre-inductive constellation $\mathbf{P}(B)$ that need not be weakly inductive (for example, any non-trivial left zero semigroup $B$ equipped with its standard order gives rise to a pre-inductive $\mathbf{P}(B)$ that fails to satisfy (I)).

Inductive constellations satisfy (O3) which involves an assertion of uniqueness, though in this case $\left(\mathrm{O} 5^{\prime}\right)$ can be replaced by the generally weaker (O5). To cover (O3), we have the following restriction-free formulation, following from Proposition 5.4. First note that in a weakly ordered constellation, if $x \leq a$ and $D(x)=e$ then $e=D(x) \leq D(a)$, so $D(x) \cdot D(a)$ exists by the second part of Proposition 5.5, whence so does $e \cdot a$. 
Corollary 5.9 The constellation $P$ is inductive if and only if it is weakly inductive and satisfies the law

$$
x \leq a \text { and } D(x)=e \text { imply } e \cdot a=x .
$$

In the pre-inductive case, if $e, f \in D(P)$ then $e \leq f$ implies $e=e|f=f| e$ by $\left(\mathrm{I}^{\prime}\right)$.

Proposition 5.10 Let $P$ be a pre-inductive constellation. For $e, f \in D(P), e \leq f$ if and only if $e=f \mid e$.

Proof. If $e \leq f$ then $e=f \mid e$ by $\left(\mathrm{I}^{\prime}\right)$. Conversely, if $e=f \mid e$ then $e \leq f$ by (O4).

Proposition 5.11 Every pre-inductive constellation is a co-restriction constellation.

Proof. We prove the laws in turn. (R1) has a proof formally identical to the proof of Lemma 3.2 in [7], applied to the case in which $b=e \in D(P)$ (making use only of (O4)). If $e \mid f=f$ then $f \leq e$ by (O4), and so $f=f \mid e$ by Proposition $5.5(2)$, proving (R2). For (R3), note that $e \mid f=f$ implies $f \leq e$ by (O4), so $(s \mid e)|f=s| f$ by Proposition 5.5 (3). For (R4), $s \mid e \leq s$, so $D(s \mid e) \leq D(s)$, and so $D(s \mid e)=D(s) \mid D(s \mid e)$ by Proposition 5.10. (R5) is just $\left(\mathrm{O} 5^{\prime}\right)$.

So all notions for co-restriction constellations may be applied directly to preinductive constellations also.

Proposition 5.12 Suppose $P$ is a pre-inductive constellation. Then $\mathbf{T}(P)$ is an ordered left congruence $D$-semigroup when equipped with the order on $P$. If $P$ is weakly inductive, then $\mathbf{T}(P)$ is ordered left Ehresmann, and if $P$ is inductive then $\mathbf{T}(P)$ is left restriction equipped with its natural order $(a \leq b$ if and only if $a=D(a) b)$.

Proof. The pre-inductive constellation $P$ is a co-restriction constellation by Proposition 5.11. Hence $\mathbf{T}(P)$ is a left congruence D-semigroup by Proposition 4.5.

Compatibility of $\leq$ with respect to $\otimes$ is an in [7] (Proposition 4.4). Moreover, for $a \in P$ and $e \in D(P), a \otimes e=(a \mid e) \cdot e=a \mid e \leq a$. Monotonicity of $D$ simply carries over. If $P$ is weakly inductive, then it is D-semiadequate, so $\mathbf{T}(P)$ is ordered left Ehresmann by Proposition 4.5. If $P$ is inductive then $\mathbf{T}(P)$ is left restriction equipped with its natural order as follows from Proposition 4.5 in [7].

We define ordered radiants between weakly ordered constellations to be co-radiants that additionally respect the order (generalising Definition 3.8 in [7]).

Again, the proof of the following is very similar to that of Theorem 4.13 and prior results in [7].

Theorem 5.13 The category of ordered left congruence D-semigroups is isomorphic to the category of pre-inductive constellations and ordered radiants. 
Proof. Propositions 5.12, 5.11 and 4.6 establish a one-to-one correspondence between the objects of these two categories. It remains to show that morphisms correspond. However, it was already established in Theorem 4.8 that co-radiants between co-restriction constellations correspond to left congruence D-semigroup homomorphisms. So the correspondence of morphisms follows since the ordering is unchanged in passing from constellation to D-semigroup and vice versa.

Corollary 5.14 The category of ordered left Ehresmann D-semigroups is isomorphic to the category of weakly inductive constellations and ordered radiants.

The left restriction semigroup/inductive constellation correspondence may be viewed as a specialisation of this last result. In this special case, $\left(\mathrm{O}^{\prime}\right)$ is specialised to (O3), and correspondingly some arbitrary order on a left Ehresmann D-semigroup that is compatible with multiplication and $D$ is specialised to the natural order on a left restriction semigroup.

\section{Open problems}

In [14], Lawson's main result is to establish a correspondence between Ehresmann categories and two-sided Ehresmann semigroups. There is interest in generalising this result to cover the case of two-sided Ehresmann semigroups equipped with a partial order not determined by the algebraic structure (for example the semigroup of binary relations on a set equipped with its fundamental order of set inclusion). These should correspond to ordered categories satisfying weakened versions of the Ehresmann category axioms. Such a result would be a "two-sided" version of Corollary 5.14 above.

\section{Acknowledgements}

The author gratefully acknowledges Professor Victoria Gould for the many helpful discussions whilst he was on sabbatical at the University of York in mid-2014, and further interactions since. The current work may be viewed as part of a larger collaboration with Professor Gould on the subject of constellations.

\section{References}

[1] Batbedat, A.: $\gamma$-demi-groups, demi-modules, produit demi-directs. In Semigroups, Proceedings, Oberwolfalch, Germany 1978, Lecture Notes in Mathematics 855, Springer-Verlag, pp. 1-18 (1981)

[2] Berberian. S.K.: Baer rings and Baer *-rings. The University of Texas at Austin, Registered U.S. Copyright Office March 1988, revised edition. (2003)

[3] Branco, M.J.J., Gomes, G.M.S., Gould, V.: Left adequate and left Ehresmann monoids. Internat. J. Algebra Comput. 21, 1259-1284 (2011) 
[4] Bredikhin, D.A.: Abstract characterisation of some classes of binary relation algebras. Algebra and Number Theory No. 2, pp. 3-19. Kabardino-Balkarsk. Gos. Univ., Nalchik, 1977 [In Russian]

[5] Fountain, J, Gomes, G.M.S., Gould, V.: A Munn type representation for a class of E-semiadequate semigroups. J. Algebra 218, 693-714 (1999)

[6] Gomes, G.M.S., Gould, V.: Left adequate and left Ehresmann monoids II. J. Algebra 348, 171-196 (2011)

[7] Gould, V., Hollings, C.: Restriction semigroups and inductive constellations. Comm. Algebra 38, 261-287 (2009)

[8] Gould, V., Hollings, C.: Partial actions of inverse and weakly left E-ample semigroups. J. Australian Math. Soc. 86, 355-377 (2009)

[9] Gould, V., Stokes, T.: Constellations and their relationship to categories. To appear in Algebra Universalis

[10] Grätzer, G.: Universal Algebra. 2nd Edition, Springer, New York (1979)

[11] Jackson, M., Stokes, T.: An invitation to C-semigroups. Semigroup Forum 62, 279-310 (2001)

[12] Jackson, M., Stokes, T.: Agreeable semigroups. J. Algebra 266, 393-417 (2003)

[13] Kambites, M.: Retracts of trees and free left adequate semigroups, Proc. Edinburgh Math. Soc. 54, 731-747 (2011)

[14] Lawson, M.V.: Semigroups and ordered categories I: the reduced case. J. Algebra 141, 422-462 (1991)

[15] Maeda, S.: On the lattice of projections of a Baer *-ring. J . Sci. Hiroshima Univ. Ser. A 22, 75-88 (1958)

[16] Manes, E: Guarded and banded semigroups. Semigroup Forum 72, 94-120 (2006)

[17] Möller, B., Struth, G.: Algebras of modal operators and partial correctness. Theoret. Comp. Sci. 351, 221-239 (2006)

[18] Resende, P.: Étale groupoids and their quantales. Adv. Math. 208, 147-209 (2007)

[19] Stokes, T.: Domain and range operations in semigroups and rings. Comm. Algebra 43, 3979-4007 (2015)

[20] Trokhimenko, V.S.: Mengers function systems. Izv. Vysš. Učebn. Zaved. Matematika 11, 71-78 (1973) [In Russian] 